\title{
Original
}

\section{Avances sobre la influencia del tejido adiposo en el adenocarcinoma de próstata}

\author{
Constanza López Fontana, M. Eugenia Maselli Artola, M. Cristina Vanrell Rodríguez, \\ Nicolás A. Di Milta Mónaco, Rafael Pérez Elizalde, José D. López Laur*
}

Laboratorio de Enfermedades Metabólicas y Cáncer. Facultad de Farmacia y Bioquímica.

Universidad Juan Agustín Maza. Mendoza. Argentina. *Cátedra Clínica Urológica. Facultad de Ciencias Médicas.

Universidad Nacional de Cuyo. Mendoza. Argentina

\begin{abstract}
Resumen
Numerosos estudios han intentado interpretar la asociación entre la obesidad y el cáncer de próstata (CaP), aunque los resultados no han sido concluyentes debido a que existe una gran dificultad para evaluar sus efectos de sobre el desarrollo del CaP. Dicha dificultad radica en que no solo implica un exceso de grasa corporal, sino también una alteración de diversos parámetros fisiológicos que aumentan la agresividad del tumor.

Objetivos: Realizar una puesta al día sobre el rol del tejido adiposo en el desarrollo del CaP. Material y métodos: se realizó una búsqueda bibliográfica y lectura compresiva de artículos relacionados con "tejido adiposo", "obesidad", "adipoquinas" y "cáncer de próstata" en Pubmed y revistas científicas.

Resultados: Estudios recientes indican que la obesidad influiría en la carcinogénesis prostática mediante factores promotores (andrógenos, leptina, VEGF, IL-6, insulina e IGF-1) y factores protectores (adiponectina e IGFBP-3). Los mecanismos potenciales mediante los cuales la obesidad podría iniciar, promover o favorecer la progresión del CaP son los bajos niveles de testosterona y altos niveles de estrógenos, el síndrome metabólico coexistente, el aumento en la secreción de leptina, VEGF, IL-6 y TNF- $\alpha$ y disminución de adiponectina, y la excesiva ingesta de grasas saturadas.

Conclusiones: El exceso de tejido adiposo en el organismo podría promover la progresión del CaP, más que ser un factor de riesgo. Sin embargo, futuros estudios son necesarios para esclarecer aún más la relación entre las adipoquinas y el CaP con el fin de desarrollar nuevas medidas de prevención y tratamiento de este tumor.
\end{abstract}

Palabras clave: Tejido adiposo. Adipoquinas. Obesidad, Cáncer de próstata.

Advances on the influence of adipose tissue on prostate cancer

\section{Abstract}

Numerous studies have investigated the association between obesity and prostate cancer (CaP), although the results have not been concluding due to the great difficulty to evaluate the effects of obesity on the development of this type of tumor. The aim of this study was to carry out a comprehensive over-view of the existing evidence about the role of adipose tissue in the prostate carcinogenesis. Recent evidence suggests that androgens, leptin, IL-6, VEGF, insulin and IGF-1 may play a role in PC progression, while adiponectin and IGFBP-3 may act as "anti- prostatic cancer" adipokines. The potential mechanisms by which obesity may initiate, promote or facilitate the progression of CaP are low levels of testosterone and high levels of estrogen, coexisting metabolic syndrome, increased secretion of leptin, VEGF, IL-6 and TNF- $\alpha$ and decreased adiponectin, and excessive intake of saturated fat.

Conclusion: Obesity may promote the progression of established PC rather than being a risk factor for the development of this tumour. However, additional studies are needed to clarify the relationship between adipokines and PC before developing new preventive or treatment strategies for this tumor.

Keywords: Adipose tissue. Adipokines. Obesity. Prostate cancer.

$\mathrm{E}$ 1 cáncer es una de las causas más importantes de morbimortalidad en el mundo y, en particular, el adenocarcinoma de próstata $(\mathrm{CaP})$ es la segunda causa de muerte por enfermedades neoplásicas en los hombres mayores de 50 años ${ }^{1}$. En las últimas dos décadas, la tasa de incidencia de esta patología ha aumentado en forma dramática en Europa constituyendo uno de los principales retos actuales para la salud pública ${ }^{2}$.

El CaP, al igual que la mayoría de los tumores malignos, tiene un periodo latente de 10 a 20 años, el cual provee un tiempo amplio para realizar pre- 
vención. En la actualidad, existe evidencia de que factores endógenos y exógenos ejercen influencia sobre la carcinogénesis prostática ${ }^{3,4}$. Entre ellos la obesidad, definida como una excesiva acumulación de energía en forma de tejido adiposo en el organismo, actuaría tanto como un factor de riesgo en la etapa de iniciación así como un factor promotor en la etapa de progresión ${ }^{5}$. El tejido adiposo no sólo es considerado un reservorio pasivo de energía, sino también un auténtico órgano de gran actividad endocrina y metabólica ${ }^{6}$ capaz de afectar el desarrollo de esta neoplasia (Fig. 1).

El incremento de la prevalencia mundial tanto del CaP como de la obesidad ha orientado parte de la investigación actual hacia la asociación entre ambas patologías. Diversos estudios epidemiológicos han mostrado una relación directa entre la obesidad y el $\mathrm{CaP}^{7,8}$ aunque otros trabajos no han podido demostrar dicha asociación ${ }^{9}$. Estudios más recientes indican que la obesidad promocionaría el desarrollo de un CaP más agresivo resultando en una tasa de recurrencia mayor después del primer tratamiento ${ }^{10}$ y una tasa de mortalidad más alta ${ }^{5,11}$. Sin embargo, hasta el momento, los resultados no han sido concluyentes debido a que existe una gran dificultad para evaluar los efectos de la obesidad sobre el desarrollo de este tipo de tumor. Dicha dificultad radica en que la obesidad no sólo se presenta como un exceso de grasa corporal, sino que también modifica diversos parámetros fisiológicos que aumentan la agresividad del $\mathrm{CaP}^{5,12-14}$ tal como se muestra en la Figura 2.

- Alteración de la secreción de adipoquinas: se incrementa la liberación de leptina, VEGF, IL-6 y TNF$\alpha$ y disminuye la secreción de adiponectina. Las adipoquinas podrían estar ligadas a diferentes mecanismos carcinogénicos incluyendo diferenciación celular, apoptosis, proliferación celular, angiogénesis y alteración de los niveles de hormonas sexuales esteroideas ${ }^{8}$.
- Inflamación crónica con secreción elevada de sustancias como el factor de necrosis tumoral alfa (TNF- $\alpha$ ) y la interleuquina 6 (IL-6) relacionados, a su vez, con el desarrollo de ciertos tumores. El TNF- $\alpha$ induce la liberación de IL-6 y leptina e interfiere en la vía de señalización de la insulina desencadenando la resistencia insulínica (RI) que se presenta en la obesidad ${ }^{15,16}$. Por su parte, la RI con hiperinsulinemia favorece un incremento de la producción hepática de factor de crecimiento similar a insulina circulante (IGF-1). Estudios recientes sugieren que niveles elevados de insulina e IGF-1 actuarían como factores promotores del $\mathrm{CaP}^{17,18}$. Específicamente, se ha observado que el IGF1 in vitro estimula el crecimiento de líneas celulares prostáticas andrógeno independientes al ejercer un efecto mitógeno y antiapoptótico sobre estas células, mientras que in vivo el IGF-1 se comporta como un factor de crecimiento de múltiples tumores malignos ${ }^{19}$.

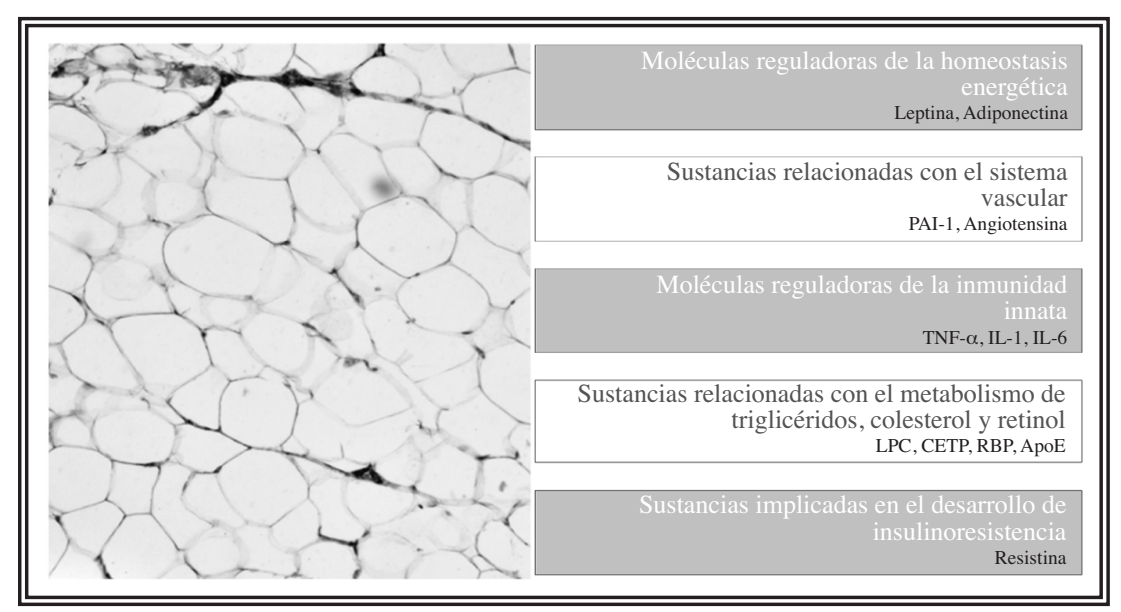

FIGURA 1. Sustancias secretadas por el tejido adiposo blanco.

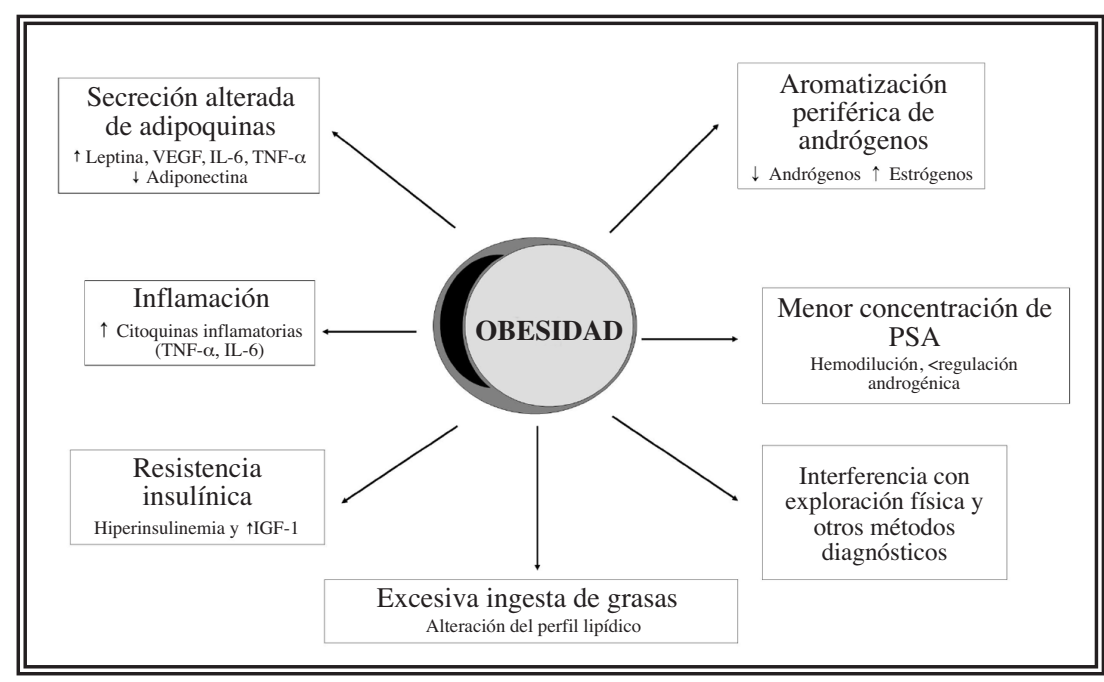

FIGURA 2. Influencia de la obesidad sobre el cáncer de próstata. 
- Mayor aromatización de andrógenos debido a una sobreexpresión de la P450 aromatasa en el tejido adiposo aumentando los niveles de estrógenos circulantes y disminuyendo los de andrógenos. Este "disbalance hormonal esteroideo sexual" ha sido asociado a tumores avanzados y de pobre diferenciación al momento de ser diagnosticados ${ }^{4,20}$.

- Menor concentración plasmática de antígeno prostático específico (PSA) con respecto a los hombres normopeso; posiblemente, debido a una menor actividad androgénica y/o a un mayor volumen plasmático (hemodilución). Esta menor concentración de PSA dificulta el diagnóstico y detección precoz del CaP en obesos, ya que se ha comprobado que muchos pacientes que al momento del screening tenían un valor de PSA dentro del intervalo normal (menor a 4ng/ml), desarrollan luego cánceres muy agresivos y tardiamente detectados ${ }^{21}$. Además, la obesidad interfiere en la exploración física y en los métodos de diagnóstico complementarios tales como tacto rectal, ecografias, y biopsias eco-dirigidas dificultando aun más la detección precoz y el diagnóstico del $\mathrm{CaP}^{21}$.

- Ingesta de alimentos hipercalóricos y ricos en grasas saturadas que alteran el perfil lipídico e influyen en el desarrollo del $\mathrm{CaP}^{22}$.

En sintesis, la obesidad es un factor de riesgo significativo para el desarrollo de CaP, aunque los mecanismos exactos de esta relación aún necesitan ser determinados ${ }^{23}$. Numerosos trabajos de investigación indican que el tejido adiposo y las diferentes sustancias que éste secreta podrían promover o prevenir el desarrollo del CaP. Las hormonas sexuales, la leptina, el VEGF, la IL-6, la insulina y el IGF-1 tendrían un efecto promotor del tumor; mientras que la adiponectina y la IGFBP-3 serían factores protectores (Fig. 3) ${ }^{1,13}$.

El presente constituye un artículo de actualización escrito con el fin de facilitar la comprensión de la influencia que ejerce el tejido adiposo sobre el CaP.

Para la redacción del mismo, se llevó a cabo una búsqueda bibliográfica en Pubmed y revistas científicas utilizando los siguientes términos para la selección de los artículos de interés: "obesidad", "adipoquinas” y “cáncer de próstata”. Se realizó la lectura comprensiva de aquellos artículos de reciente publicación y que fueron considerados apropiados con al objeto del presente escrito y se discutieron y analizaron los más relevantes.

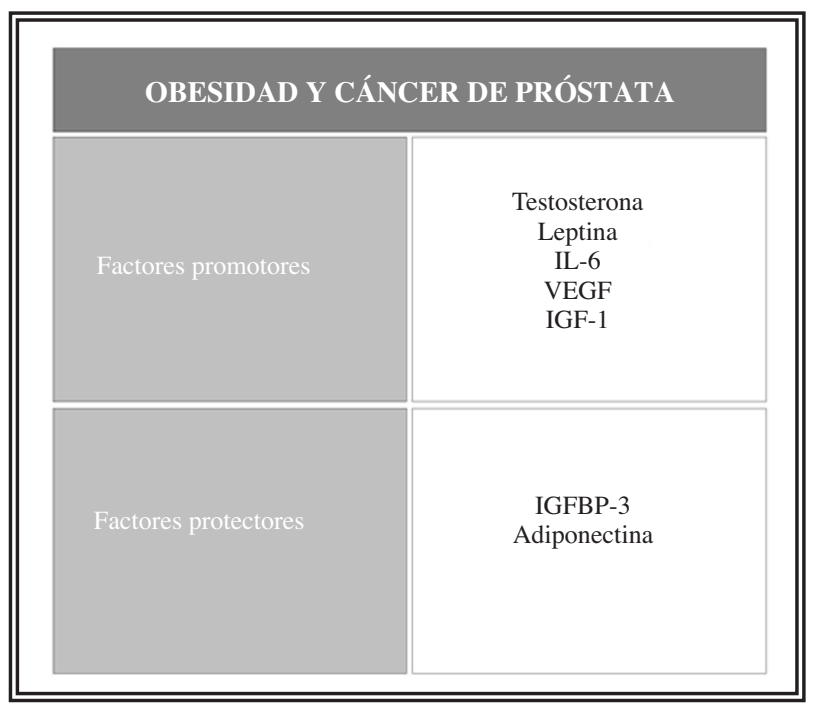

FIGURA 3. Factores promotores y protectores del cáncer de próstata.

\section{Andrógenos}

El rol de los andrógenos y su influencia en el desarrollo, maduración y mantenimiento del CaP es controversial $^{24}$. La ausencia de una exposición prolongada a los andrógenos parece proteger del CaP, pero no ha sido establecida aún una relación dosisrespuesta entre las concentraciones de andrógenos y el riesgo de cáncer. Un meta análisis prospectivo no encontró diferencias significativas entre la concentración plasmática de andrógenos de controles y pacientes con $\mathrm{CaP}^{25}$, mientras que otros estudios muestran que hombres con altas concentraciones plasmáticas de testosterona total y libre tienen menor riesgo de desarrollar CaP de alto grado ${ }^{26}$.

La máxima estimulación de crecimiento del CaP es alcanzada con relativamente bajas concentraciones de testosterona, desarrollándose así el concepto de "saturación"27.

El receptor androgénico media la actividad de la testosterona y la dihidrotestosterona (DHT) proveniente de la acción de la 5 alfa-reductasa. Ambas formas hormonales son capaces de activar el receptor androgénico y a través de él juegan un papel importante en el desarrollo y la función de la próstata $^{28,29}$.

A través de la DHT principalmente, los andrógenos inducen mitosis y apoptosis. La DHT se une al receptor androgénico aumentando la traslocación del complejo esteroide-receptor en el núcleo y activa los elementos de respuesta a los andrógenos. Teóricamente, y luego de muchos años de divisiones celulares pueden acumularse mutaciones espontáneas 
en las células prostáticas que aumentarían el riesgo de desarrollar $\mathrm{CaP}^{30}$. Algunos polimorfismos de SRD5A2 y la sustitución de alanina por treonina en el codón 49 pueden llevar a un mal pronóstico. También se piensa que algunos genes que intervienen en la síntesis de testosterona podrían inducir a la carcinogénesis ${ }^{31}$.

En definitiva, el rol preciso de los andrógenos en la carcinogénesis prostática es aún poco claro. Tradicionalmente, se ha pensado que la testosterona provoca el crecimiento del CaP motivo por el cual es contraindicada en pacientes con este diagnóstico, y también en individuos con riesgo de desarrollarlo. Sin embargo, estudios recientes han desmitificado esta relación haciendo más permisivo el uso de la hormona ${ }^{4,32}$.

\section{Leptina}

La leptina es un polipéptido de 167 aminoácidos con un peso molecular de $16 \mathrm{kD}$ y es producida principalmente en el tejido adiposo blanco. La leptina está involucrada en la regulación del balance energético a través de sus efectos centrales sobre el apetito, y periféricos sobre el gasto energético. Además, actúa en el metabolismo de los lípidos y los glúcidos y modula procesos inmunológicos, hematopoyéticos e incluso reproductivos ${ }^{33}$.

La concentración de leptina circulante se correlaciona positivamente con la grasa corporal total, el estado nutricional del individuo y el contenido de triglicéridos de los adipocitos, por lo que niveles séricos de leptina son elevados en individuos obesos comparados con individuos de peso normal, actuando de esta manera como un "marcador" de las reservas energéticas del organismo ${ }^{34}$.

Los receptores de leptina (ob-R) consisten en una proteína de membrana conformada por tres dominios: extracelular, transmembrana e intracelular. Se han observado al menos seis isoformas las cuales se encuentran distribuidas en diferentes regiones del hipotálamo y en distintos tejidos periféricos como pulmón, riñón, hígado, músculo esquelético, tejido adiposo, testículos, islotes pancreáticos y células hematopoyéticas ${ }^{33}$

Las células cancerígenas prostáticas poseen receptores de leptina y se ha observado que tanto in vitro como in vivo ésta estimula el crecimiento de líneas andrógeno-independientes y promueve la angiogénesis y proliferación de células vasculares, produciendo de esta manera la progresión, invasión y metástasis de tumores prostáticos ${ }^{35}$. Aunque, cabe aclarar que las células prostáticas cancerígenas andrógeno-dependientes no son influenciadas por la leptina en su crecimiento ${ }^{36}$.

\section{Factor de crecimiento epitelial vascular}

$\mathrm{El}$ factor de crecimiento epitelial vascular (VEGF) es una proteína implicada en la vasculogénesis (formación de novo del sistema circulatorio embrionario) y en la angiogénesis (crecimiento de vasos sanguíneos provenientes de vasos preexistentes) que ejerce sus funciones a través de dos receptores del tipo tirosín quinasa FLK/ KDR y Flt-137.

Las acciones del VEGF han sido estudiadas en las células del endotelio vascular, aunque también se han observado efectos sobre otros tipos celulares. Por ejemplo, estimula la migración de monocitos/macrófagos, neuronas, células epiteliales renales y células tumorales de diferentes tejidos ${ }^{13}$.

Las concentraciones séricas de VEGF se encuentran positivamente correlacionadas con la masa grasa visceral, ya que este factor es producido también por el tejido adiposo ${ }^{13}$. Estudios recientes han observado una correlación directa entre los niveles plasmáticos de VEGF y el estadio, el grado, la agresividad y las manifestaciones clínicas del $\mathrm{CaP}^{38,39}$. Se ha demostrado un aumento de la densidad vascular, por incremento de la angiogénesis, en el tejido canceroso respecto al tejido normal, correlacionándose con otros marcadores pronósticos del $\mathrm{CaP}$. Este incremento de la angiogénesis se ha correlacionado con CaP de alto grado de Gleason y estadios avanzados (pT3) ${ }^{39}$. Por lo tanto, el VEGF juega un rol crucial en la progresión del CaP, y es posible que las células prostáticas cancerígenas se encuentren influenciadas por la secreción aumentada de este factor en los estados de obesidad ${ }^{13}$.

\section{IL- 6}

La IL-6 es una citoquina multifuncional producida por diferentes tipos celulares, incluyendo las células del sistema inmune, células endoteliales, fibroblastos, miocitos y tejido adiposo blanco ${ }^{33}$.

La IL-6 estaría implicada en la modulación de la función inmune y la regulación de las diferentes funciones celulares incluyendo proliferación, apoptosis, angiogénesis y diferenciación. Su concentración en suero es directamente proporcional a la obesidad visceral y a la RI y niveles séricos de IL-6 superiores a $7 \mathrm{pg} / \mathrm{ml}$ están asociados a un peor pro- 
nóstico de CaP. Además, los niveles incrementados en tumores órgano- confinados sugieren un rol de la IL-6 en el estadio temprano de la carcinogénesis prostática $^{13}$.

Estudios en líneas celulares andrógeno-independientes demostraron que la IL-6 actúa como un regulador paracrino y autocrino del crecimiento del Cap. El incremento de la IL-6 relacionado con la obesidad podría potencialmente interferir y mejorar este mecanismo y promover aún más la carcinogénesis de la próstata ${ }^{36,40}$. Por lo tanto, la IL-6 actuaría en la iniciación y progresión del tumor siendo directamente proporcional al tiempo de exposición, es decir, al tiempo que el individuo lleve siendo obeso $^{36}$.

También se ha observado la existencia de un polimorfismo en el gen de la IL-6 que estaría fuertemente asociado a la agresividad y recurrencia del CaP, sugiriendo que existiría mayor riesgo de padecer un CaP recurrente debido a diferencias genéticas en el gen humano de la IL-6 ${ }^{41}$.

\section{Insulina}

La obesidad, en particular la de tipo central, está asociada con hiperinsulinemia, RI y diabetes mellitus. Se ha sugerido que los niveles elevados de insulina actuarían como un factor de crecimiento para el $\mathrm{CaP}$, resultando en un incremento del riesgo de padecer el mismo ${ }^{1,42,43}$. Sin embargo, los datos obtenidos hasta el momento son controversiales. Algunos investigadores sugieren que la diabetes incrementa el riesgo de padecer $\mathrm{CaP}^{35}$, mientras que otros equipos de investigación sostienen que está asociada a un menor riesgo de padecerlo ${ }^{44,45}$.

\section{IGF-1}

El factor de crecimiento símil insulina de tipo 1 (IGF-1) es un factor polipeptídico de 70 aminoácidos con un peso molecular de 7650 Da. Posee un 50\% de similitud estructural con la proinsulina y funciones similares a la insulina. Circula en la sangre unido a un grupo de proteínas de alta afinidad (IGFBPs 1 a 6).

In Vitro, el IGF-1 ha demostrado estimular el crecimiento de lineas celulares prostáticas andrógenoindependientes 36,46 siendo una de las tantas hormonas polipeptídicas que tiene efectos mitogénicos y antiapoptóticos sobre las células epiteliales prostáticas $^{47}$. In vivo, el IGF-1 es un factor de crecimiento de múltiples tipos de tumores malignos. Sus elevadas concentraciones en suero en obesos podrían ser uno de los mecanismos que permite asociar la obesidad a la mortalidad por diversos tipos de neoplasias, incluido el $\mathrm{CaP}^{1}$.

El incremento de la concentración de IGF-1 está directamente relacionado con factores dietarios como la ingesta de grasas poliinsaturadas, y se encuentra inversamente relacionado con elementos dietarios protectores como los vegetales, especialmente el tomate ${ }^{48}$. Esta relación permite fortalecer la asociación entre la dieta y el CaP.

Algunos estudios han encontrado que la obesidad se encuentra asociada a elevadas concentraciones libres de IGF-1 bioactiva por la disminución de su proteína transportadora IGFBP-3. Otros estudios epidemiológicos ajustados por el índice de masa corporal (IMC), encontraron una correlación directa y positiva en las concentraciones séricas de IGF-1 y el riesgo de desarrollar $\mathrm{CaP}$. Y finalmente, múltiples trabajos han observado que a mayores concentraciones de IGFBP-3 hay menores niveles de IGF-1 libre, lo que se asocia a un menor riesgo de $\mathrm{CaP}^{47}$.

\section{Adiponectina}

La adiponectina es una proteína formada por 244 aminoácidos con un peso molecular de $30 \mathrm{kDa}$ y es producida exclusivamente por el tejido adiposo blan$\mathrm{co}^{13}$. Se han caracterizado y secuenciado dos subtipos de receptores de adiponectina adR1 y adR2.

A diferencia de otras adipoquinas, los niveles circulantes de adiponectina son inversamente proporcionales a la obesidad (particularmente la central), IMC, acumulación de grasa visceral y RI ${ }^{13}$. De hecho, se ha demostrado que el descenso de peso inducido por dieta aumenta los niveles de ARNm de adiponectina en el tejido adiposo abdominal, así como sus niveles plasmáticos circulantes ${ }^{33}$. Una reducción de los niveles de adiponectina en plasma también ha sido observada en obesos con diabetes mellitus tipo 2 , hipertensión y síndrome metabólico ${ }^{13}$.

Debido a estas propiedades opuestas de la adiponectina respecto de otras adipoquinas, se la ha caratulado como la adipoquina "anticáncer" incluyendo el $\mathrm{CaP}^{49}$. Estudios recientes han observado que los niveles plasmáticos de adiponectina son significativamente menores en sujetos con CaP respecto a los que tienen hiperplasia benigna prostática y los casos control ${ }^{23}$. Además, estos estudios también observaron asociación negativa entre adiponectina, Gleason y estadío de la enfermedad ${ }^{5}$. 
En resumen, existe evidencia que sugiere una asociación entre la obesidad y el desarrollo del CaP ya que ha sido descrito que el exceso de tejido adiposo en el organismo podría promover la progresión del CaP, más que ser un factor de riesgo. Las adipoquinas tienen un rol estimulante sobre las células cancerosas prostáticas induciendo la promoción y progresión del tumor. Sin embargo, futuros estudios son necesarios para esclarecer aun más esta relación y de esta manera establecer el desarrollo de nuevas medidas de prevención, detección precoz y tratamiento del CaP.

Financiación de la investigación: Universidad Juan Agustín Maza y Fundación Allende. Las instituciones sólo proporcionaron ayuda financiera sin tener influencia en el estudio.

\section{REFERENCIAS}

1. Freedland SJ, Aronson WJ. Obesity and prostate cancer. Urology. 2005;65(3):433-439.

2. Marberger M. Prostate cancer 2008: challenges in diagnosis and management. Editorial European Urology Supplements 2009; 8:89-96

3. Chan JM, Gann PH, Giovannucci EL. Role of diet in prostate cancer development and progression. J Clin Oncol. 2005;23(32): 8152-8160.

4. Mearini L, Costantini E, Zucchi A, Mearini E, Bini V, Cottini E, Porena M. Testosterone levels in benign prostatic hypertrophy and prostate cancer. Urol Int. 2008;80(2):134-140.

5. O`Malley RL, Teneja SS. Obesity and prostate cancer. Can J Urol. 2006;13(2):11-17.

6. Irrigaría P, Newby JA, Lacomme S, Belpomme D. Overweight/ obesity and cancer genesis: more than a biological link. Biomed Pharmacoter. 2007;61(10):665-678.

7. Presti JC Jr. Obesity and prostate cancer. Curr Opin Urol. 2005; 15(1):13-16.

8. Baillargeon J, Rose DP. Obesity, adipokines, and prostate cancer. Int J Oncol. 2006;28(3):737-745.

9. Freedland SJ, Sokoll LJ, Mangold LA, Bruzek DJ, Mohr P, Yiu SK, et al. Serum leptin and pathological findings at the time of radical prostatectomy. J Urol. 2005;173(3):773-776.

10. Magheli A, Rais-Bahrami S, Trock BJ, Humphreys EB, Partin AW, Han M, et al. Impact of Body Mass Index on Biochemical Recurrence rates after radical prostatectomy: an analysis utilizing propensity score matching. Urology. 2008;72(6):12461251.

11. Amling CL. Relationship between obesity and prostate cancer. Curr Opin Urol. 2005;15(3):167-171.

12. Freedland SJ, Platz EA. Obesity and prostate cancer: making sense out of apparently conflicting data. Epidemiol Rev 2007; 29: 88-97.

13. Mistry T, Digby J, Desai K, Randeva H. Obesity and Prostate Cancer: A role for Adipokines. European Association of Urology. 2007;52(1):46-53.

14. Freedland JS, Terris MK, Platz EA, Priesti JC Jr. Body mass index as a predictor of Prostate Cancer; development versus detection on biopsy. Urology. 2005;66(1):108-113.
15. Bouraoui Y, Ricote M, García-Tuñón I, Rodriguez-Berriguete G, Touffehi M, Rais NB, et al. Pro-inflammatory cytokines and prostate-specific antigen in hyperplasia and human prostate cancer. Cancer Detect Prev. 2008;32(1):23-32.

16. Danforth KN, Rodriguez C, Hayes RB, Sakoda LC, Huang WY, Yu K, et al. TNF polymorphisms and prostate cancer risk. Prostate. 2008;68(4):400-407.

17. Rowlands MA, Gunnell D, Harris R, Vatten LJ, Holly JM, Martin RM. Circulating insulin-like growth factor peptides and prostate cancer risk: A systematic review and meta-analysis. Int J Cancer. 2008. Epub ahead of print.

18. Nandeesha H, Koner BC, Dorairajan LN. Altered insulin sensitivity, insulin secretion and lipid profile in non-diabetic prostate carcinoma Acta Physiol Hung. 2008;95(1):97-105.

19. Frasca F, Pandini G, Sciacca L, Pezzino V, Squatrito S, Belfiore A, et al. The role of insulin receptors and IGF-I receptors in cancer and other diseases. Arch Physiol Biochem. 2008;114(1): 23-37.

20. Gustafsson O, Norming U, Gustafsson S, Eneroth P, Aström G, Nyman CR. Dihydrotestosterone and testosterone levels in men screened for prostate cancer: a study of a randomized population. Br J Urol. 1996;77(3):433-440.

21. Bañez LL, Hamilton RJ, Partin AW, Vollmer RT, Sun L, Rodríguez C, et al. Obesity-related plasma hemodilution and PSA concentration among men with prostate cancer. JAMA. 2007;298(19):2275-2280.

22. Buschemeyer WC, Freedland SJ. Obesity and prostate cancer: epidemiology and clinical implications. Eur Urol. 2007;52(2): 331-343.

23. Housa D, Housova J, Vernerova Z, Haluzik M. Adipocytokines and cancer. Physiol Res. 2006;55(3):233-244.

24. Imamoto T, Suzuki H, Yano M, Kawamura K, Kamiya N, Araki $\mathrm{K}$, et al. The role of testosterone in the pathogenesis of prostate cancer. Int J Urol. 2008;15(6):472-480.

25. Eaton NE, Reeves GK, Appleby PN, Key TJ. Endogenous sex hormones and prostate cancer: a quantitative review of prospective studies. Br J Cancer. 1999 80(7):930-934.

26. Platz EA, Giovannucci E. The epidemiology of sex steroid hormones and their signaling and metabolic pathways in the etiology of prostate cancer. $J$ Steroid Biochem Mol Biol. 2004;92(4):237-253.

27. Marks LS, Mazer NA, Mostaghel E, Hess DL, Dorey FJ, Epstein JI, et al. Effect of testosterone replacement therapy on prostate tissue in men with late-onset hypogonadism: a randomized controlled trial. JAMA. 2006;296(19):2351-2361.

28. Metrebian S. Tumores de Próstata. In: López Laur JD (eds) Manual de Urologia Moderna $1^{\text {ra }}$ Ed. Buenos Aires: Atlante, 2004, p 317-335.

29. Bain J. The many faces of testosterone. Clin Inter Aging. 2007; $2(4): 205-18$

30. Correa Ochoa JJ. Testosterona y su relación con el cáncer de próstata. Urología Colombiana; 2006: 77-84

31. Stanbrough M, Bubley GJ, Ross K, Golub TR, Rubin MA, Penning TM, et al. Increased expression of genes converting adrenal androgens to testosterone in androgen-independent prostate cancer. Cancer Res. 2006;66(5):2815-2825.

32. Kokontis JM, Hay N, Liao S. Progression of LN-CaP prostate tumor cells during androgen deprivation: Hormone-independent growth, regression of proliferation by androgen and role for p27Kip1 in androgen-induced cell cycle arrest. Mol Endocrinol. 1998;12(7):941-953.

33. Moreno JC, Martínez JA. Tejido adiposo: órgano de almacenamiento y órgano secretor. An Sist Sanit Navar. 2002;25:29-39.

34. Saglam D, Aydur E, Yilmaz I, Goktas S. Leptin influences cellular differentiation and progression in prostate cancer. J Urol. 2003;169(4): 1308-1311. 
35. Gade-andavolu R, Cone LA, Shu S, Morrow A, Kowshik B, Andavolu MV. Molecular interactions of leptin and prostate cancer. Cancer J. 2006;12(3):201-206.

36. Onuma M, Bub JD, Rummel TL, Iwamoto Y. Prostate cancer cell-adipocyte interaction: leptin mediates androgen-independent prostate cancer cell proliferation through c-Jun NH2-terminal kinase. J Biol Chem. 2003;278(43):42660-42667.

37. Abdollahi A, Lipson KE, Sckell A, Zieher H, Klenke F, Poerschke $\mathrm{D}$, et al. Combined therapy with direct and indirect angiogenesis inhibition results in enhanced antiangiogenic and antitumor effects. Cancer Res. 2003;63(24):8890-8898.

38. Green MM, Hiley CT, Shanks JH, Bottomley IC, West CM, Cowan RA, et al. Expression of vascular endothelial growth factor (VEGF) in locally invasive prostate cancer is prognostic for radiotherapy outcome. Int $\mathrm{J}$ Radiat Oncol Biol Phys. 2007;67 (1):84-90.

39. Delongchamps NB, Peyromaure M. The role of vascular endothelial growth factor in kidney and prostate cancer. Can J Urol. 2007; 14(5):3669-3677.

40. Cabrespine A, Bay JO, Verrelle P, Morel L. Interleukin-6 implication in prostate cancer. Bull Cancer 2007; 94(7 Suppl): F29 34.

41. Culig Z. Interleukin-6 polymorphism is associated with more aggresive prostate cancer. J.Urol. 2005;174(2):417.

42. Hsing AW, Sakoda LC, Chua S Jr. Obesity, metabolic syndrome, and prostate cancer. Am J Clin Nutr. 2007;86(3):s843-857.

43. Smith MR, Lee H, Fallon MA, Nathan DM. Adipocytokines, obesity, and insulin resistance during combined androgen blockade for prostate cancer. Urology. 2008;71(2):318-22.
44. Will JC, Vinicor F, Calle EE. Is diabetes mellitus associated with prostate cancer incidence and survival?. Epidemiology. 1999;10(3):313-318.

45. Giovannucci E, Rimm EB, Stampfer MJ, Colditz GA, Willett WC. Diabetes mellitus and risk of prostate cancer (United States). Cancer causes control. 1998;9(1):3-9.

46. Iwamura M, Sluss PM, Casamento JB, Cockett AT. Insulin-like growth factor I: action and receptor characterization in human prostate cancer cell lines. Prostate. 1993;22(3):243-252.

47. Latif Z, Mcmillan DC, Wallace AM, Sattar N, Mir K, Jones G, et al. The relationship of circulating insulin-like growth factor I, its binding protein-3, prostate-specific antigen and C-reactive protein with disease stage in prostate cancer. BJU International. 2002;89(4):396-399.

48. Mucci LA, Tamimi R, Lagiou P, Trichopoulou A, Benetou V, Spanos E, et al. Are dietary influences on the risk of prostate cancer mediated though the insulin-like growth factor system? BJU International. 2001;87(9):814-820.

49. Kelesidis I, Kelesidis T, Mantzoros CS. Adiponectin and cancer: Systematic review. Br J Cancer. 2006;94:1221-1225.

Correspondencia autor: Dra. Constanza López Fontana Acceso Este 2245 (Lateral Sur)

San José, Guaymallén. Mendoza

Argentina. CP: 5519. Fax: 54-261-4299350

E-mail autor: investigacioncap@yahoo.com.ar.

Información artículo: Original - Cáncer de próstata

Trabajo recibido: octubre 2008

Trabajo aceptado: noviembre 2008 